\title{
Translocation of polysialic acid across model membranes: Kinetic analysis and dynamic studies ${ }^{\star \star}$
}

\author{
Teresa Janas ${ }^{1}$, Henryk Krajiński ${ }^{2}$, Anna Timoszyk ${ }^{2}$ and Tadeusz Janas ${ }^{2,3 凶}$ \\ ${ }^{1}$ Department of Physics, Technical University, Zielona Góra, Poland, ${ }^{2}$ Department of Biophysics, \\ Pedagogical University, Zielona Góra, Poland, ${ }^{3}$ Institute of Physics, Technical University, Wroctaw, \\ Poland
}

Received: 20 November, 2000; revised: 7 February, 2001; accepted: 9 March, 2001

Key words: polysialic acid, membrane transport, polyanion flux, polycation flux, electrical equivalent circuit, ${ }^{1}$ H-NMR, lipid bilayers

\begin{abstract}
Transmembrane translocation of polyion homopolymers takes place in the case of polyanionic polysialic acid (polySia), polyanionic polynucleotides and polycationic polypeptides. The purpose of this work was to determine the role of membrane electrical parameters on the kinetics of polyion translocation, the influence of polysialic acid on ion adsorption on positively charged membrane surface and the dynamics of the phospholipid hydrocarbon chains and choline group by using ${ }^{1}$ H-NMR. The analysis of polyion translocation was performed by using the electrical equivalent circuit of the membrane for the initial membrane potential equal to zero. The changes in polysialic acid flux was up to $75 \%$ after $1 \mathrm{~ms}$ in comparison with the zero-time flux. Both a decrease of membrane conductance and an increase of polyion chain length resulted in the diminution of this effect. An increase of praseodymium ions adsorption to positively charged liposomes and an increase of the rate of segmental movement of the $-\mathrm{CH}_{2}$ and $-\mathrm{CH}_{3}$ groups, and the choline headgrup of lipid molecules, was observed in the presence of polySia. The results show that the direction of the vectorial polyion translocation depends both on the membrane electrical properties and the degree of polymerization of the polymer, and that polysialic acid can modulate the degree of ion adsorption and the dynamics of membrane lipids.
\end{abstract}

The transmembrane translocation of polysialic acid chains across the inner membrane of Escherichia coli $\mathrm{K} 1$ cells requires both the membrane protonmotive force and the transmembrane electrical potential gradient [1, 2]. Polysialic acid (polySia) chains are linear homopolymers (the de-

\footnotetext{
${ }^{\star}$ Presented at the 36th Meeting of the Polish Biochemical Society, Poznań, 2000.

This work was carried out within the research project No. 6 PO4A 01410 supported by the State Committee for Scientific Research in 1996-1998 (KBN, Poland). The financial support of the UNESCO/PAS Polish Network for Cell and Molecular Biology to Mrs. Anna Timoszyk is gratefully acknowledged.

${ }^{凶}$ Author for correspondence: Tadeusz Janas, Department of Biophysics, Pedagogical University, Monte Cassino 21 B, 65-561 Zielona Góra, Poland; tel.: (48 68) 323 4080, fax: (48 68) 326 5449, e-mail: tjanas@asia.aw.wsp.zgora.pl

Abbreviations: polySia, polysialic acid; DP, degree of polymerization; PC, phosphatidylcholine; FT-NMR, Fourier transform nuclear magnetic resonance; FID, free induction decay.
} 
gree of polymerization $D P=$ about 8 to 200 ) of $\alpha$-2,8-linked sialic acid [3]. The valence of this polyanion corresponds to the degree of polySia polymerization. The minimum chain length of polySia reflects the formation of a characteristic helical conformation [4]. The genes encoding proteins necessary for the synthesis and expression of the polySia capsule in $E$. coli $\mathrm{K} 1$ have been cloned and characterised [5]. In E. coli, the 12 to 14 genes required for these processes are located in the multiple kps cluster. The $17 \mathrm{~kb} k p s$ cluster is divided into three functional regions [6]. The central region 2 contains the information for the synthesis, activation and polymerization of sialic acid. Region 3 genes are postulated to be involved in the transport of polySia across the bacterial inner membrane while region 1 genes appear to function in the transport of polymers to the external surface of the outer membrane. In eukaryotic cells, polySia functions as an oncodevelopmental antigen in human kidney and brain, and may enhance the metastatic potential of Wilms tumour cells and neuroblastomas [7]. These novel carbohydrate chains also appear to have a regulatory role in cell growth, differentiation, fertilisation and neuronal pathogenicity [7]. Studies on the function of polySia suggest that its primary role is to promote developmentally controlled and activity-dependent plasticity in cell interactions and thereby facilitate changes in the structure and function of the nervous system [8].

Vectorial translocation of negatively charged polyionic DNA across the cell membrane into the recipient cell takes place at an early stage of genetic transformation, transfection, viral infection and conjugation. Bacterial as well as plant and animal cells are able to bind nucleic acids and take them up [9]. Electrotransfection of cells, i.e., transfection induced by electric field pulses is an effective technique for introduction of foreign nucleic acid into cells [10-12].

Ohm's law and the equivalent circuit approach were used by Hodkin and Huxley to derive a relationship between total membrane current membrane capacitance, membrane voltage, time, the ion and leakage conductance, the equilibrium potentials for each of the ions, which they applied to nerve signalling [13]. Attempts to modify the
Hodkin-Huxley equation have met with limited success. Mullens and Noda added a factor to the Goldman equation [14] in order to include the effects of an ion pump on the membrane potential. Modifications of the Hodkin-Huxley equation have been applied to solve for membrane voltage in the presence of electrogenic pumps [15].

In the present paper an analysis of transmembrane translocation of polyion homopolymers was performed by using the method of the electrical equivalent circuit of the membrane and the first step of the translocation process ion adsorption on the membrane surface - was studied by using ${ }^{1}$ H-NMR technique. The theoretical study presents the dependence of the polyanion or polycation flux through membrane on time, membrane electrical conductance, membrane electrical capacitance, the degree of polymerisation, water solution conductance and the transmembrane potential applied. ${ }^{1}$ H-NMR technique was used to study the effect of polySia on praseodymium ions desorption from liposomes and the dynamics of the phospholipid hydrocarbon chains and choline group.

\section{MATERIALS AND METHODS}

Calculations. Figure 1 presents the electrical equivalent circuit of the membrane. Passive diffusion of ions across the membrane is represented by the left-hand branch of the circuit: the membrane resistance $\left(G_{d}{ }^{-1}\right)$ is connected in series with the electromotive force $\left(E_{d}\right)$ which is equivalent to the diffusion potential. Electrogenic pumps, which actively transport ions out of the cell, are represented by the next (going right) branch: $G_{p}$ is the total conductance of the electrogenic pumps located in the membrane; $E_{p}$ is the electromotive force of the electrogenic pumps. $C_{m}, G_{s}$ and $V(t)$ represent the membrane capacitance, the electrical conductance of the bathing solution and the electrodes, and the external potential source, respectively. The switch, $S$, closes the circuit at time $t=0$.

From the first Kirchhoff's law and Ohm's law we have: 


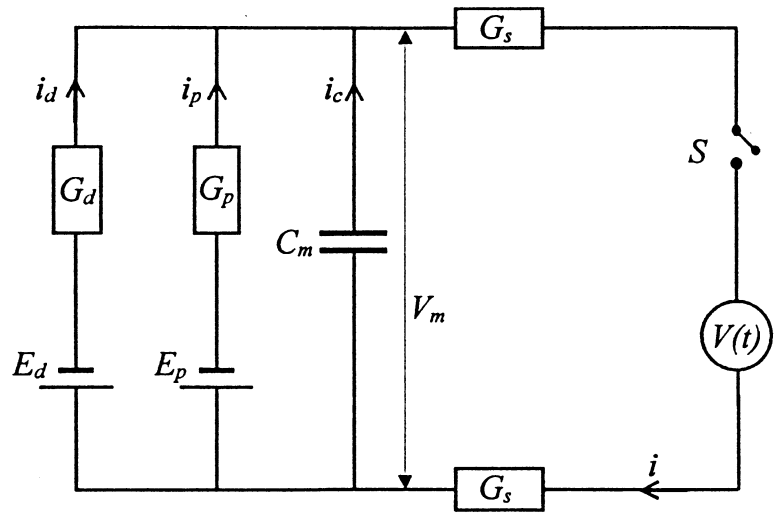

Figure 1. Equivalent electrical circuit of a biomembrane.

Where: $G p\left[\Omega^{-1} \times \mathrm{cm}^{-2}\right]$ - electrical conductance of the ion pump; $G_{d}\left[\Omega^{-1} \times \mathrm{cm}^{-2}\right]$ - electrical conductance of the membrane for the diffusion process; $G_{s}\left[\Omega^{-1} \times \mathrm{cm}^{-2}\right]$ - electrical conductance of the bathing solution and the electrodes; $E_{d}[\mathrm{~V}]$ - diffusion potential; $E_{p}[\mathrm{~V}]$ - electromotive force of ion pumps; $i\left[\mathrm{~A} \times \mathrm{cm}^{-2}\right]-$ the total current; $i_{d}\left[\mathrm{~A} \times \mathrm{cm}^{-2}\right]$ - diffusive current; $i_{p}\left[\mathrm{~A} \times \mathrm{cm}^{-2}\right]-$ current passing through the pumps; $i_{c}\left[\mathrm{~A} \times \mathrm{cm}^{-2}\right]-$ capacitive current per surface unit; $V_{m}$ [V] - electrical potential of the membrane; $C_{m}\left[\mathrm{~F} \times \mathrm{cm}^{-2}\right]$ - capacitance of the membrane; $V(t)[\mathrm{V}]$ - external potential source. The switch, $S$, closes the circuit at the time $t=0$.

$i=i_{d}+i_{p}+i_{c}$

$i d=G_{d}\left(V_{m}-E_{d}\right)$

$i_{p}=G_{p}\left(V_{m}-E_{p}\right)$

$i_{c}=C_{m} \frac{d V_{m}}{d t}$

where $i\left[\mathrm{~A} \times \mathrm{cm}^{-2}\right]$ is the total current, $i_{d}$ is the diffusive current, $i_{p}$ is the current passing through the pumps, $i_{c}$ is the capacitive current (all currents per unit area), $t$ [s] is time, $G_{d}\left[\Omega^{-1} \times \mathrm{cm}^{-2}\right]$ is the electrical conductance of the membrane resulting from the diffusion process, $E_{d}[\mathrm{~V}]$ is the diffusion potential, $G_{p}\left[\Omega^{-1} \times \mathrm{cm}^{-2}\right]$ is the electrical conductance of ion pumps, $E_{p}[\mathrm{~V}]$ is the electromotive force of ion pumps, $C_{m}\left[\mathrm{~F} \times \mathrm{cm}^{-2}\right]$ is the capacitance of the membrane, $V_{m}[\mathrm{~V}]$ is the electrical potential of the membrane.

After combining we get:

$$
i=C_{m} \frac{d V_{m}}{d t}+V_{m}\left(G_{d}+G_{p}\right)-\left(G_{d} E_{d}+G_{p} E_{p}\right)
$$

In the case when the membrane potential, $V_{m}$, equals to the resting potential, $E_{r}$, the current passing through the membrane $i_{r}=i_{d}+i_{p}$. Therefore:

$E_{r} G_{m}=G_{d} E_{d}+G_{p} E_{p}$

where $G_{m}\left[\Omega^{-1} \times \mathrm{cm}^{-2}\right]$ is the electrical conductance of the membrane, $G_{m}=G_{d}+G_{p}$.

The resting state of the membrane $\left(V_{m}=E_{r}\right)$ is represented by the switch, $S$, in the "open circuit" position.

Combining Eqns. (2) and (3) we obtain:

$i=C_{m} \frac{d V_{m}}{d t}+V_{m} G_{m}-E_{r} G_{m}$

From the second Kirchhoff's law:

$V_{m}=V-\frac{2 i}{G_{s}}$

where $V[V]$ is the external potential; $G_{s}$ $\left[\Omega^{-1} \times \mathrm{cm}^{-2}\right]$ is the electrical conductance of the bathing solution and the electrodes, $G_{s}=1 / R_{s}=$ $i / V_{s} ; V_{s}[V]$ is the drop of the electrical potential on the electrical resistance, $R_{s}[\Omega]$, of the bathing solution and electrodes.

Combining Eqns. (4) and (5) we obtain:

$i+\frac{2 G_{m} i}{G_{s}}+\frac{2 C_{m}}{G_{s}} \times \frac{d i}{d t}=C_{m} \frac{d V}{d t}+G_{m} V-E_{r} G_{m}$

Since $i=J z F$, where $J$ [mole $\times \mathrm{s}^{-1} \times \mathrm{cm}^{-2}$ ] is ion flux through the membrane, $z$ is the ion valence, $F=9.65 \times 10^{4}\left[\mathrm{C} \times \mathrm{mol}^{-1}\right]$ is the Faraday's constant, we obtain:

$z F J+\frac{2 z F G_{m}}{G_{s}} J+\frac{2 C_{m} z F}{G_{s}} \times \frac{d J}{d t}=$

$=C_{m} \frac{d V}{d t}+G_{m} V-E_{r} G_{m}$

After substituting for

$\alpha=z F+\frac{2 z F G}{G_{s}}$ and $\beta=\frac{2 C_{m} z F}{G_{s}}$

we get:

$\alpha \cdot+\beta \frac{d J}{d t}=G_{m} V-E_{r} G_{m}+C_{m} \frac{d V}{d t}$ 
Equation (9) represents a first order differential equation. This first order differential equation can be solved for $V=0$ (the electrical potential source, $V(t)$, keeps the external potential, $V$, at 0 ) for the initial condition, $J(t=0)=J_{0}$ :

$J(t)=-\frac{G_{m} E_{r}}{\alpha}+\left[J_{0}+\frac{G_{m} E_{r}}{\alpha}\right] e^{-\frac{\alpha}{\beta} t}$

Equation (10) describes the relationship between unidirectional flux of polyions across the membrane and the variables membrane conductance, membrane capacitance, polyion degree of polymerisation and time.

Chemicals. Egg-yolk phosphatidylcholine (PC), praseodymium chloride $\left(\mathrm{PrCl}_{3} \times 6 \mathrm{H}_{2} \mathrm{O}\right)$ and polysialic acid (polySia) were purchased from Sigma. Octadecylamine $\left(\mathrm{CH}_{3}\left(\mathrm{CH}_{2}\right)_{17} \mathrm{NH}_{2}\right)$ was from Fluka. Heavy water $\left(\mathrm{D}_{2} \mathrm{O}\right.$, purity $\left.99.9 \%\right)$ was obtained from the Institute of Nuclear Research (Świerk, Poland).

Preparation of unilamellar liposomes. Phosphatidylcholine was mixed with octadecylamine in chloroform, dried under nitrogen and dispersed in $\mathrm{D}_{2} \mathrm{O}$. The final concentration of phosphatidylcholine was $25 \mathrm{mg}$ per $\mathrm{ml}$. The constant concentration of octadecylamine was $5.0 \mathrm{~mol} \%$ with respect to the lipid. Polysialic acid (polySia) was mixed with liposome suspension at a final concentration of $0.0,0.25,1.0$ or $5.0 \mathrm{mg}$ per ml, then sonicated for $30 \mathrm{~min}$ with a $20 \mathrm{kHz}$ sonicator with a titanium probe.

${ }^{1}$ H-NMR experiments. In Fourier transform (FT)-NMR experiments, the signal from excited nuclei is observed following the pulse via voltage changes (nuclear induction) in a coil around the sample tuned to the resonance frequency. This signal decreases in intensity to zero with time as the nuclei return, or relax, to their original state of thermal equilibrium. Hence, the signal is termed the free-induction decay (FID). Fourier transform of the FID, or a summation of FIDs, yields a conventional absorption-type spectrum (the Lorentzian line). The intensity of the signal from a population of identical nuclei is linearly proportional to the population size [16, 17].

It is useful to identify two components of nuclear relaxation. One is termed spin-spin, or trans- verse, relaxation, by which energy is transferred from one nucleus to another. In homogeneous liquids, or in complex systems where there are strong interactions between different types of nuclei, this relaxation process can be described by a simple exponential decay, characterised by a time constant, $T_{2}$. The spin-spin relaxation time, $T_{2}$, characterises the decay of the FID from the population of identical nuclei in a pulse experiment. Such inhomogeneity results in nuclei in different portions of the sample precessing at different frequencies, since they experience different field strengths, so that the phase of one nucleus relative to others necessarily changes. Hence, if inhomogeneity effects are significant, the time constant for the decay of the FID from an assembly of identical nuclei is $T_{2}$ [18]. It can readily be seen that as $T_{2}$ increases, the half-width of the resonance signal $\Delta v_{1 / 2}$ gets narrower, in fact:

$\Delta v_{1 / 2}=\left(\pi T_{2}\right)^{-1}$

Since $T_{2}$ reflects how long neighbouring nuclei take to exchange magnetic energy with their environment, this parameter is approximately proportional to the rate of molecular motion. On the basis of this theory, it follows that rapid molecular motions produce sharp spectral lines, while atoms in environments where motion is restricted exhibit substantially broader signals [19, 20]. Line half-width can also be influenced by chemical shift exchange processes [17, 21]. The second relaxation process is termed spin-lattice, thermal or longitudinal relaxation, in which energy contained in a nuclear spin system is lost to surrounding molecules (or lattice) in the form of heat (rotational and translational motion). Spin-lattice relaxation time, $T_{1}$, like spin-spin relaxation time, is also an exponential phenomenon in homogeneous liquids. Unlike $T_{2}, T_{1}$ is not influenced by magnetic field inhomogeneity [18].

NMR data were collected for samples of $0.5 \mathrm{ml}$ vesicle suspension with $5.0 \mathrm{mM} \mathrm{PrCl} 3$ in $5 \mathrm{~mm}$ NMR tubes. ${ }^{1}$ H-NMR spectra were recorded on a Bruker Avance DRX 300 spectrometer at $300 \mathrm{~K}$. The $300 \mathrm{MHz}$ spectrometer parameters were: spectral window $6172.8 \mathrm{~Hz}$; digital resolution 
$150.0 \mathrm{~Hz} / \mathrm{cm}$ or $0.5 \mathrm{ppm} / \mathrm{cm}$; pulse width $10.6 \mu \mathrm{s}$; acquisition time $2.654 \mathrm{~s}$; and delay time $1.0 \mathrm{~s}$.

\section{RESULTS AND DISCUSSION}

In this study we used the electrical equivalent circuit analysis in order to develop a biophysical model of the modulation of transmembrane translocation rates of polyanionic homopolymers by electric field. Plots, showing the flux as a function of the degree of polyion polymerization are symmetrical with respect to the symmetry line perpendicular to the $D P$ axis and passing through the point $J=J_{0}$ on the flux axis. The value of $J_{0}=$ $5 \times 10^{-17}$ mole $\times \mathrm{s}^{-1} \times \mathrm{cm}^{-2}$ (the number of moles of polySia translocated in $1 \mathrm{~s}$ per $1 \mathrm{~cm}^{2}$ of the inner membrane surface of a bacterial cell) was evaluated from the polySia biosynthesis rate [22] and from the number of polySia chains per one bacterial cell estimated in [23]. The replacement of a polyanion by a polycation of the same chain length gives the same shape of the obtained surface plot but with opposite orientation.

The relationship between the polySia flux and the membrane conductance and time is presented in Fig. 2. For the membrane conductance of $10^{-5}$ $\Omega^{-1} \times \mathrm{cm}^{-2}$ the polySia flux changes from $5 \times$ $10^{-17}$ mole $\times \mathrm{s}^{-1} \times \mathrm{cm}^{-2}(t=0)$, through the value $-7.41 \times 10^{-17}$ mole $\times \mathrm{s}^{-1} \times \mathrm{cm}^{-2}$ (the minus sign represents the flux of opposite direction), to $-5.92 \times 10^{-15}$ mole $\times \mathrm{s}^{-1} \times \mathrm{cm}^{-2}(t=$ $1 \mathrm{~s})$. This means that there is an 80-fold increase in the flux in the time interval $\left[10^{-3} ; 1\right] \mathrm{s}$. For the membrane conductance $10^{-8} \Omega^{-1} \times \mathrm{cm}^{-2}$ the changes in the polySia flux are much smaller: from the value $5 \times 10^{-17}$ mole $\times \mathrm{s}^{-1} \times \mathrm{cm}^{-2}(t=$ $0)$, through $4.98 \times 10^{-17}$ mole $\times \mathrm{s}^{-1} \times \mathrm{cm}^{-2}(t=$ $1 \mathrm{~ms})$ to $-5.99 \times 10^{-17}$ mole $\times \mathrm{s}^{-1} \times \mathrm{cm}^{-2}(t=1$ s). Figure 3 a shows polySia flux as a function of the membrane conductance and the degree of polymerization of polySia for time $t=1 \mathrm{~ms}$. For $D P=200$ the flux decreases from $4.99 \times 10^{-17}$ mole $\times \mathrm{s}^{-1} \times \mathrm{cm}^{-2}$ to $-1.26 \times 10^{-17}$ mole $\times \mathrm{s}^{-1}$ $\times \mathrm{cm}^{-2}$ with the increase of the membrane conductance from $10^{-8} \Omega^{-1} \times \mathrm{cm}^{-2}$ to $10^{-5} \Omega^{-1} \times$ $\mathrm{cm}^{-2}$. For $D P=10$ the changes are much bigger: from the $4.87 \times 10^{-17}$ mole $\times \mathrm{s}^{-1} \times \mathrm{cm}^{-2}$ to

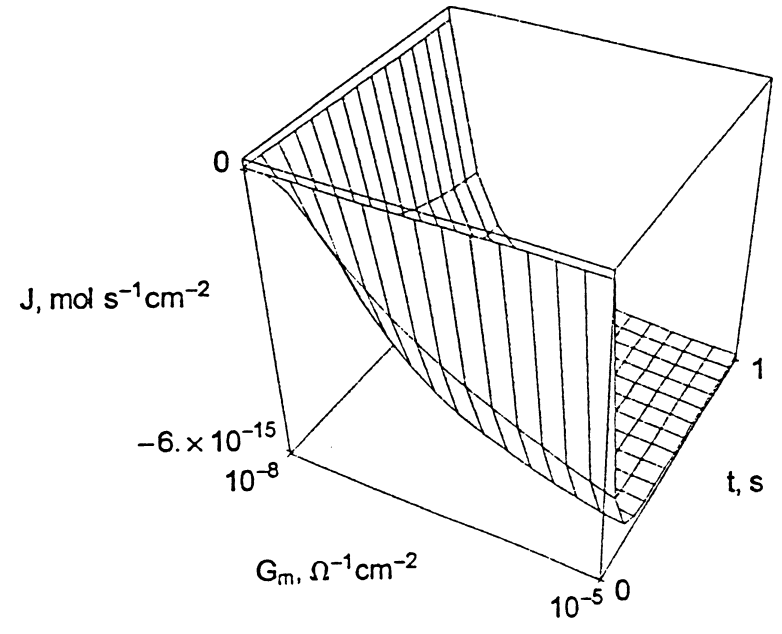

Figure 2. Dependence of the flux of polyion chains across the membrane, $J$, on the conductance of the membrane, $G_{m}$, and time, $t$.

Calculated for: resting potential of the membrane, $E_{r}=$ $-0.12 \mathrm{~V}$; electrical conductance of the bathing solution and the electrodes, $G_{s}=10^{-6} \Omega^{-1} \times \mathrm{cm}^{-2}$; capacitance of the membrane, $C_{m}=5 \times 10^{-7} \mathrm{~F} \times \mathrm{cm}^{-2}$; degree of the polyion polymerization, $D P=-100$; initial flux, $J_{0}=5 \times$ $10^{-17}$ mole $\times \mathrm{s}^{-1} \times \mathrm{cm}^{-2}$.

$-1.19 \times 10^{-15}$ mole $\times \mathrm{s}^{-1} \times \mathrm{cm}^{-2}$; for $D P=1$ the corresponding values of the flux are $3.75 \times$ $10^{-17}$ mole $\times \mathrm{s}^{-1} \times \mathrm{cm}^{-2}$ and $-1.23 \times 10^{-14}$ mole $\times \mathrm{s}^{-1} \times \mathrm{cm}^{-2}$, respectively. For the points lying below the reversal curves (Fig. 3b), the polySia flux is positive, while points above the curves give negative flux. The reversal curves were plotted for different time points.

The dependency of the polySia flux on time and the degree of polySia polymerization for the membrane conductance $10^{-6} \Omega^{-1} \times \mathrm{cm}^{-2}$ is visualised in Fig. 4a. For long polySia chains ( $D P$ equals to 200) the polySia flux changes in time from $5 \times$ $10^{-17}$ mole $\times \mathrm{s}^{-1} \times \mathrm{cm}^{-2}(t=0)$ through $4.37 \times$ $10^{-17}$ mole $\times \mathrm{s}^{-1} \times \mathrm{cm}^{-2}$ (a $12.5 \%$ decrease) to $-1.97 \times 10^{-15}$ mole $\times \mathrm{s}^{-1} \times \mathrm{cm}^{-2}$ (with the change of the flux into opposite direction) at $1 \mathrm{~ms}$ and $1 \mathrm{~s}$, respectively. For oligoSia chains $(D P=$ 10) the decrease is bigger (from $5 \times 10^{-17}$ mole $\times \mathrm{s}^{-1} \times \mathrm{cm}^{-2}$ to $-7.44 \times 10^{-17}$ mole $\times \mathrm{s}^{-1} \times$ $\mathrm{cm}^{-2}$ and $-3.94 \times 10^{-14}$ mole $\times \mathrm{s}^{-1} \times \mathrm{cm}^{-2}$, respectively). The maximal drop is observed for monomers $(D P=1)$ : from $5 \times 10^{-17}$ mole $\times \mathrm{s}^{-1} \times$ $\mathrm{cm}^{-2}$ to $-1.19 \times 10^{-15}$ mole $\times \mathrm{s}^{-1} \times \mathrm{cm}^{-2}$ and 

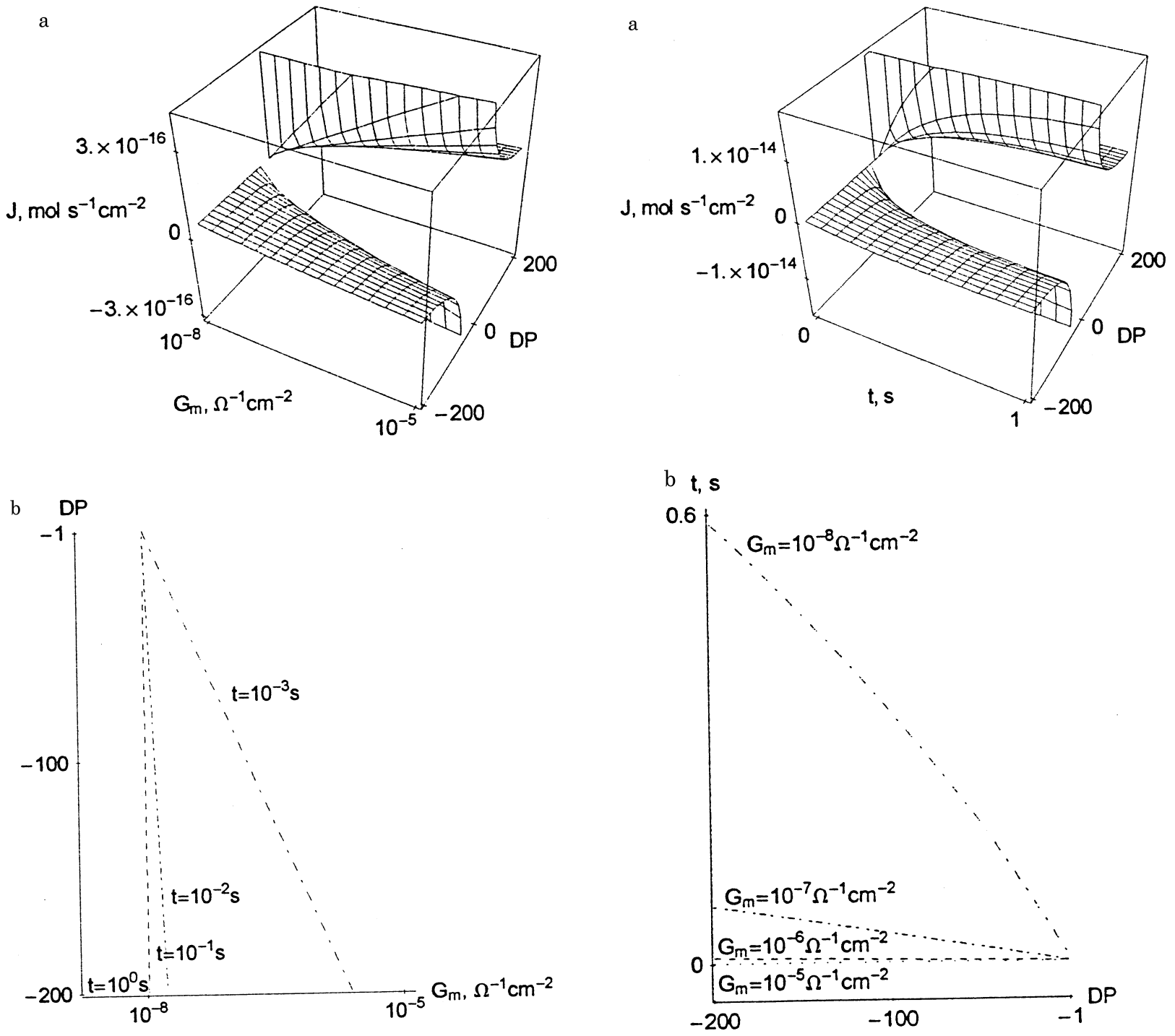

Figure 3. (a) Dependence of the flux of polyion chains across the membrane, $J$, on the conductance of the membrane, $G_{m}$, and the degree of the polyion polymerization, $D P$.

Polyanionic homopolymers are represented by negative values of $D P$ which correspond to the homopolymer ionic valence. Calculated for: resting potential of the membrane, $E_{r}=-0.12 \mathrm{~V}$; electrical conductance of the bathing solution and the electrodes, $G_{s}=10^{-6} \Omega^{-1} \times \mathrm{cm}^{-2}$; capacitance of the membrane, $C_{m}=5 \times 10^{-7} \mathrm{~F} \times \mathrm{cm}^{-2}$; time, $t=$ $10^{-3} \mathrm{~s}$; initial flux, $J_{0}=5 \times 10^{-17}$ mole $\times \mathrm{s}^{-1} \times \mathrm{cm}^{-2}$.

(b) Reversal curves, obtained from (a), showing the relationship between the degree of the polyion polymerization and the conductance of the membrane for the polyion flux $J=0$.

Each line was plotted for different time, $t$.

$-3.94 \times 10^{-13}$ mole $\times \mathrm{s}^{-1} \times \mathrm{cm}^{-2}$. The reversal curves obtained from Fig. $4 \mathrm{a}$ for different values

Figure 4. (a) Dependence of the flux of polyion chains across the membrane, $J$, on the degree of the polyion polymerization, $D P$, and time, $t$.

Polyanionic homopolymers are represented by negative values of $D P$ which correspond to the homopolymer ionic valence. Calculated for $G_{\mathrm{m}}=10^{-8} \Omega^{-1} \times \mathrm{cm}^{-2}$, the values of $E_{r}, G_{s}, C_{m}, J_{0}$ are as in Fig. 3.

(b) Reversal curves, obtained from (a), showing the relationship between time and the degree of the polyion polymerization for the polyion flux $\boldsymbol{J}=\mathbf{0}$.

The lines were plotted for different electrical conductance of the membrane, $\mathrm{G}_{m}$.

of membrane conductance are presented in Fig. 4b. The area below the curves represents positive polySia flux (in the same direction as the initial flux $J_{0}$ ) whereas the area above the curves represents negative polySia flux (in the opposite direction). 
${ }^{1}$ H-NMR spectra were recorded upon addition of polySia and $\mathrm{Pr}^{3+}$ ions to samples of phosphatidylcholine (PC)-octadecylamine vesicles. This study was undertaken in order to assess the effect of the polar groups of octadecylamine and polysialic acid on the dynamic properties of the lipid membrane. Figure 5 shows the change of distance between the choline headgroup signals coming from the outer and the inner layer of the liposome. Changes of the splitting $(\delta, \mathrm{Hz})$ between extravesicular and intravesicular choline headgroup signals is proportional to the concentration of the praseodymium added to the extravesicular

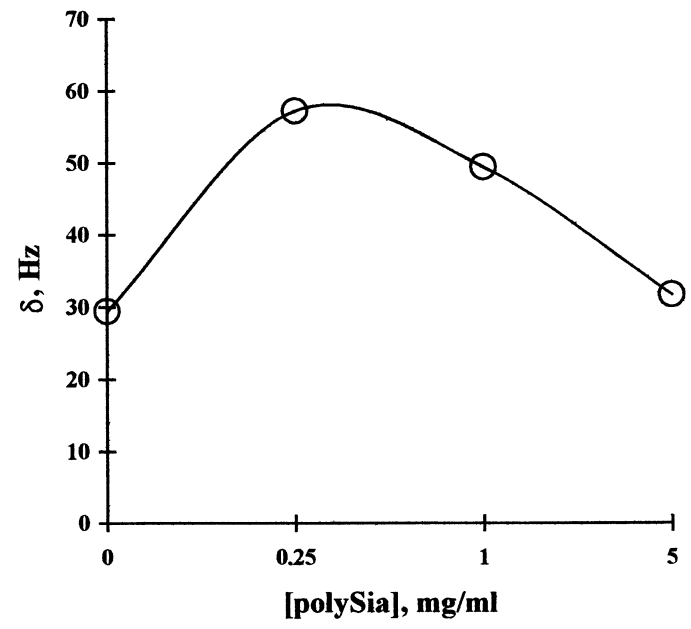

Figure 5. Increase of splitting $(\delta)$ of the maxima related to the proton resonance in $-\mathrm{N}^{+}\left(\mathrm{CH}_{3}\right)_{3}$ groups in phosphatidylcholine-octadecylamine $\quad$ 95:5, mole ratio) liposome suspension after addition of $\mathrm{PrCl}_{3}$, as a function of the concentration of polySia.

medium [8, 25, 26, 28]. Any change in the concentration of praseodymium bound to the external lipid monolayer of the liposome changes the distance (splitting) between choline headgroup signals. As seen in Fig. 5 an increase of the splitting to the maximum value for polySia concentration equal to $0.25 \mathrm{mg} / \mathrm{ml}$ and a decrease for higher concentration of polySia is observed. PolySia at a concentration of $0.25 \mathrm{mg}$ per $\mathrm{ml}$ caused minimal desorption of $\operatorname{Pr}^{3+}$ ions from the liposomes (i.e., maximal adsorption) as seen in Fig. 6. In this case the increase of adsorption of praseodymium ions was caused by the presence of polySia in the bathing solution. The percentage of praseodymium desorption was calculated as the ratio of the change in the choline headgroup signal splitting

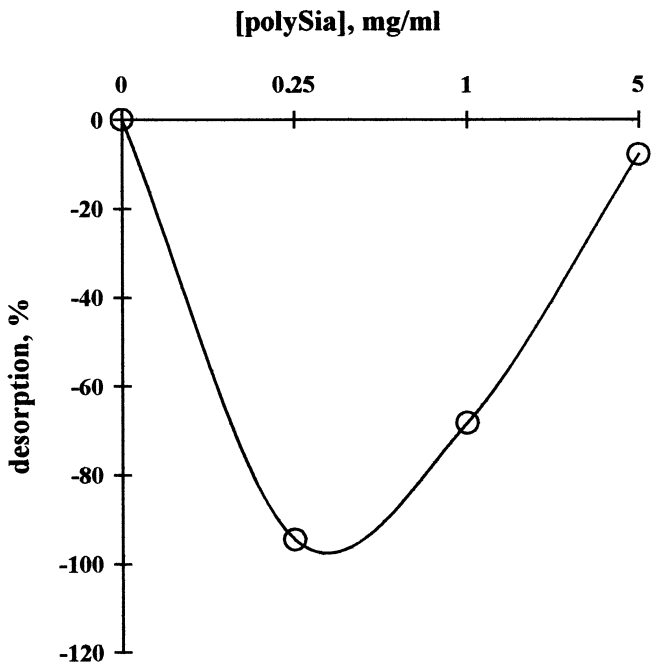

Figure 6. Percentage of $\mathrm{Pr}^{3+}$ desorption versus concentration of polySia added to liposome suspension.

The percentage of desorption was calculated as the change in the signal splitting caused by the addition of polySia to a liposome dispersion in the presence of constant extravesicular concentration of $\mathrm{Pr}^{3+}$ ions relative to the signal splitting caused by $\operatorname{Pr}^{3+}$ in the absence of polySia. The concentration of polySia was $0.0,0.25,1.0$ and $5.0 \mathrm{mg}$ per ml.

before and after addition of polySia. Figure 7 shows the polySia-related decrease of half-width $\left(\Delta v_{1 / 2}\right)$ of the $300 \mathrm{MHz}{ }^{1} \mathrm{H}-\mathrm{NMR}$ peak of choline groups corresponding to the extravesicular and the intravesicular monolayer of PC/octadecylamine liposomes. The results (Fig. 7b) show strong sharpening of spectral peaks in the intravesicular monolayer (of the liposome). The sharpening of the spectral peaks of the extravesicular monolayer is less pronounced (Fig. 7a). The effect of sharpening of spectral peaks is directly related to the unlimitation of the segmental movement of lipid molecues [19, 20]. Figure 8 shows the decreasing of half-width $\left(\Delta v_{1 / 2}\right)$ of spectral lines corresponding on $-\mathrm{CH}_{2}$ and $-\mathrm{CH}_{3}$ groups of lipid alkyl chains. The effect of sharpening of the peaks is stronger in the case of $-\mathrm{CH}_{3}$ terminal groups (Fig. 8b) than $-\mathrm{CH}_{2}$ groups (Fig. 8a). This is an indication of the fluidisation effect of polySia on the hydrophobic part of the positively-charged membrane.

Upon addition of the paramagnetic shift ion $\operatorname{Pr}^{3+}$, separate ${ }^{1} \mathrm{H}-\mathrm{NMR}$ signals can be observed from the inner and outer choline groups of the 

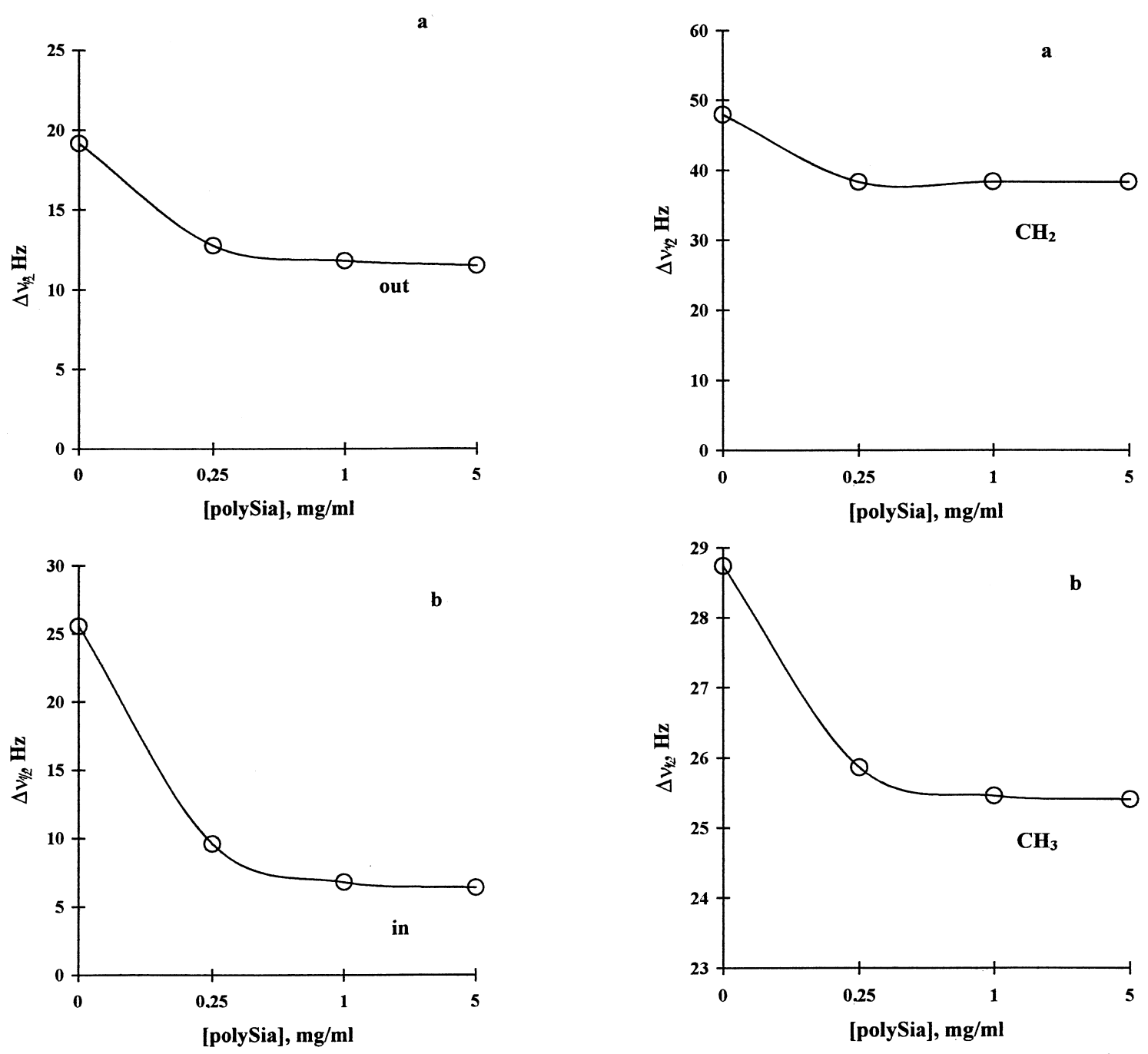

Figure 7. Decrease of half-width $\left(\Delta v_{1 / 2}\right)$ of ${ }^{1} \mathrm{H}-\mathrm{NMR}$ spectra maximum corresponding to $N$-methyl choline signals of phosphatidylcholine from the outer (a) and the inner (b) monolayer of vesicle as a function of concentration of polySia.

vesicles [24-26]. Model PC liposomes were modified with the cationic lipid octadecylamine with $\mathrm{p} K_{\mathrm{a}}$ of 10.6 which contributes a positive charge over a wide range of $\mathrm{pH}$ owing to its primary amine group [27]. The positively charged membrane caused a reduction (in comparison to neutral membranes) of adsorption of praseodymium ions to the liposomes (data not shown) which can be measured as the change of the distance between signals coming from the outer and the inner layer choline headgroups. Therefore, the positively-charged octadecylamine causes a release of $\mathrm{Pr}^{3+}$ ions from the liposomes. The addition of polySia to the extravesicular medium resulted in

Figure 8. Decrease of half-width $\left(\Delta v_{1 / 2}\right)$ of ${ }^{1}$ H-NMR spectra maximum corresponding to $-\mathrm{CH}_{2}$ groups (a) and $-\mathrm{CH}_{3}$ terminal groups (b) of phosphatidylcholine alkyl chains in the presence of polySia.

an enhanced penetration of praseodymium ions to the polar zone of the external layer of the membrane as monitored by the splitting of the $-\mathrm{N}^{+}\left(\mathrm{CH}_{3}\right)_{3}$ signal. The electrostatic interaction between praseodymium cations and the negatively charged membrane surface can induce an increase of adsorption of $\mathrm{Pr}^{3^{+}}$ions. The percentage of praseodymium desorption was calculated as the ratio of the change in the choline headgroup-signal splitting before and after addition of the compound studied [26]. A strong fluidisation effect of polySia with respect to the hydrophobic core of the positively-charged lipid bilayer was concluded from the polysialic acid dependent sharpening of the ${ }^{1} \mathrm{H}-\mathrm{NMR}$ peaks as- 
signed to $-\mathrm{CH}_{2}$ and $-\mathrm{CH}_{3}$ groups of lipid alkyl chains.

Martin and Harvey [29] applied ionic circuit analysis to examine transport in lepidopteran midgut components. In that analysis pumps, porters and channels, as well as ionic concentration gradients and membrane capacitance, were components of ionic circuits that function to transform metabolic energy (e.g., from ATP hydrolysis) into useful metabolic work (e.g., amino acid uptake). Computer-generated time courses of membrane potential reproduced key aspects of the coupling of the protonmotive force generated by an $\mathrm{H}^{+}$V-ATPase to $\mathrm{K}^{+} / 2 \mathrm{H}^{+}$antiport and amino $\mathrm{acid} / \mathrm{K}^{+}$symport in the lepidopteran midgut. A simple system consisting of a pump for a single ionic species in a membrane separating two aqueous compartments was considered [30]. That analysis departed from all previous ones by representing ionic gradients as charged compartmental capacitances instead of batteries. In the circuit, the ionic (in this case proton) pump was depicted by the pump potential/porter combination, and the frictional and slippage resistances were considered to be negligible and were ignored. In the steady state, in this case equilibrium, the pump generated a proton-motive force which was the product of the pump potential and the proton porter coupling coefficient [30]. A simple electrical equivalent circuit for the plasmalemma of cells has been shown [31]. The left-hand branch of the circuit represented passive diffusion of ions across the membrane; the membrane resistance was connected in series with the electromotive force, which was equivalent to the diffusion potential. The right-hand branch represented the electrogenic $\mathrm{H}^{+}$pump, which actively transports $\mathrm{H}^{+}$ions out of the characean cell. The steady-state diffusion of ions through separate, selective channels was described according to irreversible thermodynamics [32]. Ion fluxes thus obtained were the same as those in the parallel conductance model. The equivalent electrical circuit for this system had its electromotive forces expressed by the chemical potentials of the diffusing ions. It was concluded that the passive diffusion flows remain steady, since active transport mechanisms pump the ions up their electrochemical po- tentials. The authors of those papers focused however, on the transmembrane movement of univalent cations or anions and neglected the transport phenomena of polycations or polyanions across biological membranes.

It has been shown that DNA translocation into cells using a high-voltage pulse takes less than $3 \mathrm{~s}$, and that DNA electrophoresis has an important role in this process [33]. The authors suggest that the mechanism of cell electrotransfection is underlain by electrophoretic movement of DNA through membrane pores, the rise of which is determined by interaction with DNA in the electric field. DNA penetration from T4 phage adsorbed on $E$. coli cells was measured at different membrane potentials [34]. The authors found that the T4 DNA injection process depended on a minimum $V_{m}$ below which there was no penetration. The precipitous reduction in DNA penetration occurred between 110 and $60 \mathrm{mV}$. This threshold of membrane potential for DNA penetration was independent of $\Delta \mathrm{pH}$.

Polyprenyl phosphate functions as a transmembrane carrier during the biosynthesis of polySia in $E$. coli $\mathrm{K} 1$ [22]. The results of voltammetric analysis and Langmuir monolayer studies of model lipid membranes indicate that electrical transmembrane potential can accelerate the formation of pores in lipid bilayers modified by long chain polyprenols [35, 36]. We suggest that translocation of polySia across the inner bacterial membrane can be facilitated by pore formation in the presence of polyprenols and by transmembrane potential.

In conclusion, we have showed that transmembrane potential can modulate the transmembrane translocation rate of polyion homopolymers. The analysis describes the effect of transmembrane potential on the translocation rate of negatively charged polyanionic polysaccharides and polynucleotides across membranes. The investigations are extended to the transmembrane translocation of polycationic polypeptides. Such polypeptides strongly interact with DNA and are used to increase the uptake of foreign genes in mammalian cells with the aim to transfect them [37-39]. The results show that the direction of the vectorial polyion translocation de- 
pends both on the membrane and the degree of polymerization of the polymer. The results of 300 $\mathrm{MHz}{ }^{1} \mathrm{H}-\mathrm{NMR}$ investigations indicate that polysialic acid can modulate the dynamics of positively-charged bilayer lipid membranes and can increase the adsorption of positively-charged ions to the lipid bilayer.

The authors would like to thank Prof. Frederic A. Troy (University of California, Davis) and Dr. J. Gabrielska (Agricultural University, Wrocław, Poland) for many helpful discussions and M.Sc. U. Walkowiak from the NMR Laboratory (Technical University, Wrocław, Poland) for professional assistance with NMR measurements.

\section{R E F E R E N C E S}

1. Troy, F.A., Janas, T., Janas, T. \& Merker, R.I. (1990) Topology of the poly- $\alpha$-2,8-sialyltransferase in $E$. coli $\mathrm{K} 1$ and energetics of polysialic acid chain translocation across the inner membrane. Glycoconjugate J. 7, 383.

2. Troy, F.A., Janas, T., Janas, T. \& Merker, R.I. (1991) Vectorial translocation of polysialic acid chains across the inner membrane of Escherichia coli K1. FASEB J. 5, 6835.

3. Rutishauser, U. \& Landmesse, L. (1996) Polysialic acid in the vertebrate nervous system: A promoter of plasticity in cell-cell interactions. Trends Neurosci. 19, 422-427.

4. Michon, F., Brisson, J. \& Jennings, H. (1987) Conformational differences between linear $\alpha(2-8)$-linked homosialooligosaccharides and the epitope of the group B meningococcal polysaccharide. Biochemistry 26, 8399-8405.

5. Boulnois, G.J., Roberts, I.S., Hodge, R., Hardy, K.R., Jann, K.B. \& Timmis, K.N. (1987) Analysis of the $\mathrm{K} 1$ capsule biosynthesis genes of Escherichia coli: Definition of three functional regions for capsule production. Mol. Gen. Genet. 208, 242-246.

6. Silver, R.P., Finn, C.W., Vann, W.F., Aaronson, W., Schneerson, R., Kretchmer, P.J. \& Garon, C.F. (1981) Molecular cloning of the K1 capsular polysaccharide genes of E. coli. Nature 289, 696-698.

7. Kiss, J.Z. \& Rougon, G. (1997) Cell biology of polysialic acid. Curr. Opin. Neurobiol. 7, 640-646.
8. Rutishauser, U. (1998) Polysialic acid at the cell surface: Biophysics in service of cell interactions and tissue plasticity. J. Cell. Biochem. 70, 304-312.

9. Grinius, L. (1980) Nucleic acid transport driven by ion gradient across cell membrane. FEBS Lett. 113, $1-10$.

10. Sukharev, S.I., Klenchin, V.A., Serov, S.M., Chernomordik, L.V. \& Chizmadzhev, Y.A. (1992) Electroporation and electrophoretic DNA transfer into cells. The effect of DNA interaction with electropores. Biophys. J. 63, 1320-1327.

11. Xie, T.D., Sun, L. \& Tsong, T.Y. (1990) Study of mechanisms of electric field-induced DNA transfection. DNA entry by surface binding and diffusion through membrane pores. Biophys. J. 58, 13-19.

12. Hui, S.W., Stoicheva, N. \& Zhao, Y.L. (1996) High-efficiency loading, transfection, and fusion of cells by electroporation in two-phase polymer systems. Biophys. J. 71, 1123-1130.

13. Hodkin, A.L. \& Huxley, A.F. (1952) A quantitative description of membrane current and its application to conduction and excitation in nerve. $J$. Physiol. 117, 500-544.

14. Mullens, L.J. \& Noda, K. (1963) The influence of sodium-free solutions on the membrane potential of frog muscle fibers. J. Gen. Physiol. 47, 117-132.

15. Kishimoto, U., Kami-Ike, N. \& Takeuchi, Y. (1981) A quantitative expression of the electrogenic pump and its possible role in the excitation of Chara internodes; in: The Biophysical Approach to Excitable Systems (Adleman, W.J. \& Goldman, D.E., eds.) pp. 165-181, Plenum Press, New York.

16. Deslauriers, R. \& Smith, C.P. (1981) The multinuclear NMR approach to peptides: Structures, conformations, and dynamics; in: Biological Magnetic Resonance (Berliner, J.L. \& Reuben, J., eds.) pp. 243-344, Plenum Press, New York.

17. Roberts, J.K.M. \& Jardetzky, O. (1985) Nuclear magnetic resonance spectroscopy in biochemistry, in: Modern Physical Methods in Biochemistry (Neuberger, A. \& Deenen, L.L.M., eds.) pp. 1-64, Elsevier, New York.

18. Schuh, J.R. \& Chan, S.I. (1982) Nuclear Magnetic Resonance, in Methods of Experimental Physics; in Biophysics (Ehrenstein, G. \& Lecar, H., eds.) pp. 1 51, Academic Press, New York. 
19. Ghosh, P., Hutadilok, N. \& Adam, N. (1994) Interaction of hyaluronan (hyaluronic acid) with phospholipids as determined by gel permeation chromatography, multi-angle laser-light-scattering photometry and ${ }^{1}$ H-NMR spectroscopy. Int. J. Biol. Macromol. 16, 237-244.

20. Darke, A., Finer, E.G., Moorhouse, R. \& Rees, D.A. (1975) Studies of hyaluronate solutions by nuclear magnetic relaxation measurements. Detection of covalently-defined, stiff segments within the flexible chains. J. Mol. Biol. 99, 477-486.

21. Kuczera, J., Gabrielska, J., Kral, T.E. \& Przestalski, S. (1997) A synergistic effect of select organotin compounds and ionic surfactants on liposome membranes. Appl. Organomet. Chem. 11, 591-600.

22. Rohr, T.E. \& Troy, F.A. (1980) Structure and biosynthesis of surface polymers containing polysialic acid in Escherichia coli. J. Biol. Chem. 255, 2332-2342.

23. Weisgerber, Ch. \& Troy, F.A. (1990) Biosynthesis of the polysialic acid capsule in Escherichia coli $\mathrm{K} 1$. J. Biol. Chem. 265, 1578-1587.

24. Jones, I.C. \& Hunt, G.R.A. (1985) A ${ }^{31}$ P- and ${ }^{1}$ H-NMR investigation into the mechanism of bilayer permeability induced by the action of phospholipase $\mathrm{A}_{2}$ on phosphatidylcholine vesicles. Biochim. Biophys. Acta 820, 48-57.

25. Kaszuba, M. \& Hunt, G.R.A. (1990) ${ }^{31}$ P- and ${ }^{1}$ H-NMR investigations of the effect of n-alcohols on hydrolysis by phospholipase $\mathrm{A}_{2}$ of phospholipid vesicular membranes. Biochim. Biophys. Acta 1030, 88-93.

26. Gabrielska, J., Sarapuk, J. \& Przestalski, S. (1997) Role of hydrophobic and hydrophilic interactions of organotin and organolead compounds with model lipid membranes. Z. Naturforsch. 52c, 209 216 .

27. Piemi, M.P.Y., Korner, D., Benita, S. \& Marty, J-P. (1999) Positively and negatively charged submicron emulsions for enchanced topical delivery of antifungal drugs. J. Control. Release 58, $177-187$.

28. Gabrielska, J. \& Gruszecki, W.I. (1996) Zeaxanthin (dihydroxy- $\beta$-caroten) but not $\beta$-carotene rigidifies lipid membranes: A ${ }^{1}$ H-NMR study of carotenoidegg phosphatidylcholine liposomes. Biochim. Biophys. Acta 1285, 167-174.
29. Martin, F.G. \& Harvey, W.R. (1994) Ionic circuit analysis of $\mathrm{K}^{+} / \mathrm{H}^{+}$antiport and amino acid $/ \mathrm{K}^{+}$ symport energized by a proton-motive force in Manduca sexta larval midgut vesicles. J. Exp. Biol. 196, $77-92$.

30. Martin, F.G. (1992) Circuit analysis of transmembrane voltage relationships in V-ATPasecoupled ion movements. J. Exp. Biol. 172, 387-402.

31. Trela, Z., Janas, T., Witek, S. \& Przestalski, S. (1990) Effects of quaternary ammonium salts on membrane potential and electric conductance in internodal cells of Nitellopsis obtusa. Physiol. Plant. 78, 57-60.

32. Tomicki, B. (1999) Steady-state diffusion and the cell resting potential. Eur. Biophys. J. 28, 330-337.

33. Klenchin, V.A., Sukharev, S.I., Serov, S.M., Chernomordik, L.V. \& Chizmadzhev, Y.A. (1991) Electrically induced DNA uptake by cells is a fast process involving DNA electrophoresis. Biophys. J. 60, 804-811.

34. Labedan, B., Heller, K.B., Jasaitis, A.A., Wilson, T.H. \& Goldberg, E.B. (1980) A membrane potential threshold for phage T4 DNA injection. Biochim. Biophys. Res. Commun. 93, 625-630.

35. Janas, T., Walińska, K. \& Janas, T. (1998) Electroporation of polyprenol-phosphatidylcholine bilayer lipid membranes. Bioelectrochem. Bioenerg. 45, 215-220.

36. Janas, T., Nowotarski, K., Gruszecki, W.I. \& Janas, T. (2000) The effect of hexadecaprenol on molecular organisation and transport properties of model membranes. Acta Biochim. Polon. 47, 661-673.

37. Zenke, M., Steinlein, P., Wagner, E., Cotten, M., Beug, H. \& Birnstiel, M.L. (1990) Receptor-mediated endocytosis of transferrin-polycation conjugates: An efficient way to introduce DNA into hematopoietic cells. Proc. Natl. Acad. Sci. U.S.A. 87, 3655-3659.

38. Wymn, T.B., Nicol, F., Zelphati, O., Scaria, P.V., Plank, C. \& Szoka, F.C. (1997) Design, synthesis and characterization of a cationic peptide that binds to nucleic acids and permeabilizes bilayers. Biochemistry 36, 3008-3017.

39. Midoux, P. \& Monsigny, M. (1999) Efficient gene transfer by histidylated polylysine/pDNA complexes. Bioconjugate Chem. 10, 406-411. 